\title{
Ionization structure of hot components in symbiotic binaries during active phases
}

\author{
Z. Cariková and A. Skopal \\ Astronomical Institute, Slovak Academy of Sciences, 05960 Tatranská Lomnica, Slovakia \\ e-mail: [zcarikova; skopal]@ta3.sk
}

Received 14 March 2012 / Accepted 24 September 2012

\begin{abstract}
Context. During active phases of symbiotic binaries, an optically thick medium in the form of a flared disk develops around their hot stars. During quiescent phases, this structure is not evident.

Aims. We propose the formation of a flared neutral disk-like structure around the rotating white dwarf (WD) in symbiotic binaries. Methods. We applied the wind compression model and calculated the ionization boundaries in the compressed wind from the WD using the equation of photoionization equilibrium.

Results. During active phases, the compression of the enhanced wind from the rotating WD can form a neutral disk-like zone at the equatorial plane, while the remainder of the sphere above/below the disk is ionized. The hydrogen column density throughout the neutral zone and the emission measure of the ionized fraction of the wind, calculated for the mass loss rate from the WD, $\dot{M}=2 \times 10^{-6} M_{\odot} \mathrm{yr}^{-1}$ with $v_{\infty}=2000 \mathrm{~km} \mathrm{~s}^{-1}$, are consistent with those derived from observations. During quiescent phases, the neutral disk-like structure cannot be created because of insufficient mass loss rate.

Conclusions. Formation of the neutral disk-like zone at the equatorial plane is connected with the enhanced wind from the rotating WD, observed during active phases of symbiotic binaries. This probably represents a common origin of warm pseudophotospheres, indicated in the spectrum of active symbiotic binaries with a high orbital inclination.
\end{abstract}

Key words. binaries: symbiotic - stars: winds, outflows - stars: activity

\section{Introduction}

Symbiotic stars are interacting binary systems comprising a cool giant as the donor star and a compact star, mostly a white dwarf (WD), as the accretor. This composition constrains large orbital periods, which are typically a few years, but can be even longer. Principal process of the interaction is the mass transfer between the binary components via the stellar wind from the giant. The following accretion heats up the WD to $\gtrsim 10^{5} \mathrm{~K}$ and increases its luminosity to $\sim 10^{2}-10^{4} L_{\odot}$. Such hot and luminous WD is capable of ionizing neutral wind particles of both the stars, giving rise to strong nebular radiation. The spectrum of symbiotic stars thus consists of three main components of radiation, two stellar and one nebular. Many aspects of this general view on the nature of symbiotic stars were originally pointed by, for example, Boyarchuk (1967), Tutukov \& Yangelson (1976), Allen (1980), Seaquist et al. (1984), Kenyon \& Webbink (1984), and Nussbaumer \& Vogel (1987).

If the processes of mass loss, accretion, and ionization are in mutual equilibrium, the symbiotic system releases its energy at approximately a constant rate and spectral energy distribution (SED). This stage is called the quiescent phase. In most cases, the observed energy output of $\sim 10^{3}-10^{4} L_{\odot}$, is believed to be caused by stable hydrogen nuclear burning on the WD surface, i.e. the accreted material burns as it is accreted (e.g. Paczyński \& Rudak 1980; Fujimoto 1982). During quiescent phases, the main donor of particles into the circumbinary medium is represented by the wind from the giant. As a result, the symbiotic nebula is usually very extended, because the burning WD ionizes a large fraction of the giant's wind (Seaquist et al. 1984; Nussbaumer \& Vogel 1987). Its origin allowed the mass loss rate from the giant to be estimated to a few $\times 10^{-7} M_{\odot} \mathrm{yr}^{-1}$ (e.g. Mürset et al. 1991; Mikołajewska et al. 2002; Skopal 2005). Using the $\mathrm{H} \alpha$ method, Skopal (2006) found that the mass loss rate of the hot star during quiescent phases is significantly smaller, being of a few $\times 10^{-8} M_{\odot} \mathrm{yr}^{-1}$.

Once the equilibrium between the primary processes is disturbed, e.g. by a transient increase in the accretion rate, the symbiotic system changes its radiation significantly, brightens up in the optical by typically 1-3 mag, and shows signatures of enhanced mass outflow for a few months to years. This stage is called the active phase (e.g. Kenyon 1986). However, a precise mechanism of the outbursts is not well understood to date. Recently, Sokoloski et al. (2006) suggested that nuclear shell burning on the WD could be enhanced by the influx of material from dwarf-nova-like disk instability, while Bisikalo et al. (2006) suggested a disruption of the disk as a result of variations in the wind velocity from the giant. The SED during active phases is characterized by a relatively cool, A-F stellar type of the continuum, a decrease (or disappearance) of highly ionized lines, and an increase of the nebular hydrogen emission. Kenyon et al. (1991) and Mikołajewska \& Kenyon (1992) interpreted these features by an extended accretion disk around the hot star in CI Cyg and AX Per, whose plane is inclined to the observer with $\sim 73^{\circ}$. To avoid the problem of an unseen boundary layer, the authors speculated that the disk material expands out of the midplane and thus cools the boundary layer during the visual maximum. For the 1984-86 outburst of $\mathrm{Z}$ And, Fernández-Castro et al. (1995) also indicated a cool UV continuum with a signature of the Rayleigh scattering attenuation around Ly- $\alpha$, along with a strong nebular continuum, whose emission measure increased by a factor of 3.4 with respect to 
quiescence. They interpreted these characteristics in terms of the ejection of an optically thick shell, which substantially blocks the UV radiation. Finally, modelling the UV SED of active symbiotic binaries with a high orbital inclination confirmed the presence of such a two-temperature spectrum, where the cool component is produced by a relatively warm stellar source radiating at $\approx 20000 \mathrm{~K}$ and the hot one is represented by a strong nebular radiation (Skopal 2005). The former is not capable of producing the observed nebular emission, and thus the latter signals the presence of a hot ionizing source $\left(z 10^{5} \mathrm{~K}\right)$ in the system, which is not seen directly by the observer. In addition, a strong Rayleigh scattering attenuation of the continuum around the Ly$\alpha$ line directly indicates a significant number of neutral hydrogen atoms on the line of sight, which are not present during the quiescent phase. This effect was originally measured by FernándezCastro et al. (1995). Therefore, Skopal (2005) suggested that there is an edge-on disk around the accretor, the outer flared rim of which represents the warm pseudophotosphere. He also suggested that the nebula is placed above/below the disk, since it is ionized by the central hot star. A further important characteristic of the active phases is a significantly enhanced mass loss rate from the hot star (e.g. Fernández-Castro et al. 1995; Nussbaumer et al. 1995; Crocker et al. 2002). Modelling the broad $\mathrm{H} \alpha$ wings, Skopal (2006) found that the mass loss rate of the active hot star enhances to a few times $\left(10^{-7}-10^{-6}\right) M_{\odot} \mathrm{yr}^{-1}$, i.e. it exceeds the rate indicated during quiescent phases by a factor of $\gtrsim 10$. The ejected material is ionized by the luminous central hot star, which thus enhances radiation from the symbiotic nebula (see Tables 3 and 4 of Skopal 2005).

These general properties of quiescent and active phases thus indicate a connection between the mass loss rate from the hot star and the level of the activity of symbiotic binaries. Recently, this connection was justified by Skopal et al. (2009), who explained the anticorrelation between the supersoft X-ray and UV/optical fluxes due to the variable hot star wind during different stages of activity of the symbiotic star AG Dra.

Accordingly, in this paper we investigate the idea that the enhanced wind from the hot star can be responsible for the formation of the neutral disk-like zone during active phases. For this purpose, we applied the wind compression model, as introduced by Bjorkman \& Cassinelli (1993), to explore how the wind from the WD can be compressed towards the equatorial plane due to its fast rotation. In Sect. 2, we outline in principle the wind compression model we use in our application, and calculate its ionization structure. Section 3 compares the model with observations and discusses the results. Conclusions are found in Sect. 4.

\section{Ionization structure of the compressed wind}

Rotation of the hot star with radiation-driven winds leads to compression of the outflowing material towards the equatorial regions. The trajectory of each particle in the wind is determined by the gravity and radiation forces, which are both central forces. Due to conservation of the angular momentum vector, each particle launched at the surface of the star has to move in its own orbital plane, which is perpendicular to this vector. The mechanism can be described by the wind compression model, in which the streamlines of the gas from both hemispheres of a rotating linedriven wind are bent towards the equatorial plane (e.g. Lamers $\&$ Cassinelli 1999, and references therein). The flow of wind particles towards the equator enhances with rotation and weakens with radial distance from the star's surface. Two forms of equatorial density enhancements can be recognized: (i) if the flow from the hemispheres has a supersonic component of the velocity perpendicular to the equatorial plane, a shock zone or disk will develop. This wind-compressed disk (WCD) model was elaborated by Bjorkman \& Cassinelli (1993); (ii) if the streamlines do not cross the equator (the so-called non-crossing trajectories) we get the wind-compressed zone (WCZ) model described by Ignace et al. (1996). The shocks in the WCD model at the upper and lower boundary of the disk can produce very high temperatures (Bjorkman \& Cassinelli 1993; Krolik \& Raymond 1985). As a result, the ionization structure in the wind would be influenced by the presence of such a hot disk. In our approach, we restrict calculations for the sake of simplicity to models with non-crossing trajectories, i.e. we apply the WCZ model.

\subsection{Model assumptions}

The wind in our model begins at the surface of the hot WD with the radius $R_{\mathrm{WD}}$. It rotates with a velocity $v_{\text {rot }}$, with the rotation axis perpendicular to the orbital plane. For this paper, we adopted $R_{\mathrm{WD}}=0.01 R_{\odot}$ and $v_{\text {rot }}=100-600 \mathrm{~km} \mathrm{~s}^{-1}$ (see Sect. 2.3.1). The photons are emitted at a blackbody WD's pseudophotosphere with a temperature $T_{\star}$ and a radius $R_{\star}>$ $R_{\text {WD }}$. The pseudophotosphere represents the optically thick/thin boundary of the wind. It simulates the hot star in the model, which is characterized by the fundamental parameters $L_{\star}, R_{\star}$, and $T_{\star}$. Thus, the wind is accelerated deep inside the photosphere. As the wind mass loss rate varies between quiescent and active phases, $R_{\star}$ is also subject to variation. In the model, we assume that the WD's pseudophotosphere is spherical.

From observations, we derive the fundamental parameters $L_{\mathrm{h}}, R_{\mathrm{h}}^{\text {eff }}, T_{\mathrm{h}}$ of the hot component in the binary (i.e. the object orbiting the cool giant). These parameters can deviate significantly from those of the hot star (i.e. $L_{\star}, R_{\star}, T_{\star}$ of the WD's pseudophotosphere as defined above), depending on the level of activity and the orbital inclination. For example, the effective radius of the hot component, $R_{\mathrm{h}}^{\mathrm{eff}}$, is defined as the radius of a sphere, which has the same luminosity as the entire stellarradiating disk-like object, derived by modelling the UV SED (Skopal 2005, Eq. (19) here). During active phases, $R_{\mathrm{h}}^{\mathrm{eff}}$ can be as large as a few $R_{\odot}$ for systems with a high orbital inclination, while during quiescent phases it is clustered around $0.1 R_{\odot}$ only (e.g. Skopal 2005; Skopal et al. 2011). When viewing the system more from the pole, the effective radius shrinks significantly. An example is AG Dra, for which the mean values of $R_{\mathrm{h}}^{\text {eff }}=0.063 \pm 0.013 R_{\odot}$ and $0.033 \pm 0.010 R_{\odot}$ were derived by modelling the SED during active and quiescent phases, respectively (Skopal 2005; Skopal et al. 2009). This suggests that we measure $R_{\mathrm{h}}^{\text {eff }} \gg R_{\star}$ for eclipsing systems and $R_{\mathrm{h}}^{\text {eff }} \approx R_{\star}$ for non-eclipsing systems. Accordingly, in the model calculations we adopted $R_{\star}=0.06 R_{\odot}$ for active phases. Other parameters of the hot star and the wind that are needed for the model calculations are introduced in Sect. 3.1.

Finally, we neglect the effect of the orbital motion on the shaping of the compressed wind formation. For a typical orbital period of $\sim 2$ years and total mass of $\sim 2 M_{\odot}$ of a symbiotic binary, the orbital velocity of its components is only $v_{\text {orb }} \sim 30 \mathrm{~km} \mathrm{~s}^{-1}$. As $v_{\text {orb }}$ is significantly smaller than the terminal velocity of the wind from the WD $v_{\infty}$ (see Sect. 2.3.2), the momentum flux $\dot{M}\left(v_{\infty}+v_{\text {orb }}\right) \approx \dot{M}\left(v_{\infty}-v_{\text {orb }}\right)$ for a constant $\dot{M}$ from the WD. Therefore, the density gradient at the front and back side of the WD in the direction of its orbital motion will also be comparable, and the expected prolongation of the compressed wind formation will probably not be significant. As a result, we ignore the orbital motion of the binary and calculate the ionization structure in the compressed wind for a stationary 
situation and pure hydrogen gas, similar to the Seaquist et al. (1984) calculation of the ionization structure for the quiescent phase.

\subsection{Density in the WCZ model}

According to Bjorkman \& Cassinelli (1993), in a star-centred spherical coordinate system $(r, \theta, \phi)$, the wind compression model assumes an azimuthal symmetry, i.e. the density of the wind does not depend on the azimuthal angle $\phi$. Therefore, the density is a function of the radial distance $r$ from the star's centre and the polar angle $\theta$, measured from the spin axis. The wind density distribution follows from the mass continuity equation as

$N_{\mathrm{H}}(r, \theta)=\frac{\dot{M}}{4 \pi r^{2} \mu_{\mathrm{m}} m_{\mathrm{H}} v_{\mathrm{r}}(r)}\left(\frac{\mathrm{d} \mu}{\mathrm{d} \mu_{0}}\right)^{-1}$,

where $\dot{M}$ is the mass loss rate of the star, $\mu_{\mathrm{m}}$ is the mean molecular weight, $m_{\mathrm{H}}$ is the mass of the hydrogen atom, $v_{\mathrm{r}}(r)$ is the radial component of the wind velocity. We used the $\beta$-law wind as introduced by Lamers \& Cassinelli (1999),

$v_{\mathrm{r}}(r)=v_{\infty}\left(1-\frac{b R_{\mathrm{WD}}}{r}\right)^{\beta}$,

where $R_{\mathrm{WD}}$ is the origin of the wind, $\beta$ characterises an acceleration of the wind (i.e. how steep the velocity law is), and the parameter $b$ is given by

$b=1-\left(\frac{a}{v_{\infty}}\right)^{1 / \beta}$,

where $a$ is the initial velocity of the wind at its origin (see Sect. 2.3.2). In comparison with Bjorkman \& Cassinelli (1993), we assume that $v_{\infty}$ does not depend on the polar angle $\theta$. The geometrical factor $\mathrm{d} \mu / \mathrm{d} \mu_{0}$ describes the compression of the wind due to rotation of the star (see Bjorkman \& Cassinelli 1993; Ignace et al. 1996). A summary of the model can also be found in the textbook of Lamers \& Cassinelli (1999).

Knowing the density distribution in the compressed wind (Eq. (1)), we can determine the ionization structure using the equation of photoionization equilibrium.

\subsection{Ionization boundaries in the WCZ model}

In this section, we calculate the ionization boundaries in the wind from the hot star. The ionization boundary is defined by the locus of points at which ionizing photons are completely consumed along the path outward from the ionizing star. For the sake of simplicity, we restrict our calculations to a wind containing only hydrogen atoms and assume Case B recombination (e.g. Osterbrock 1974).

At each point of the nebula, the photoionization equilibrium equation can be written as

$N_{\mathrm{H}^{0}}(r, \theta) \int_{v_{0}}^{\infty} \frac{4 \pi J_{v}}{h v} a_{v} \mathrm{~d} v=N_{\mathrm{p}}(r, \theta) N_{\mathrm{e}}(r, \theta) \alpha_{\mathrm{B}}\left(T_{\mathrm{e}}\right)$

where $J_{v}$ is the mean intensity of radiation, $N_{\mathrm{H}^{0}}(r, \theta), N_{\mathrm{p}}(r, \theta)$ and $N_{\mathrm{e}}(r, \theta)$ are neutral hydrogen, proton and electron densities by number per unit volume, respectively, $a_{v}$ is the ionization cross section for hydrogen, $h$ is the Planck's constant, $v_{0}$ is the ionization frequency for hydrogen atoms, and $\alpha_{\mathrm{B}}\left(T_{\mathrm{e}}\right)$ is the total hydrogenic recombination coefficient in case $\mathrm{B}$.
The left-hand side of Eq. (4) gives the number of ionizations and the right-hand one gives the number of recombinations per unit volume per unit time. Their equivalence expresses the condition for the ionization equilibrium. If the absorption and geometrical dilution are taken into account, the mean intensity of the stellar radiation can be written as

$4 \pi J_{v}=4 \pi W(r) B_{v}\left(T_{\star}\right) \mathrm{e}^{-\tau_{v}}$,

where $\pi B_{v}\left(T_{\star}\right)$ is the flux at the surface of the hot star (i.e. the WD's pseudophotosphere, see Sect. 2.1), $\tau_{v}$ is the radial optical depth for hydrogen, and $W(r)$ is the geometrical dilution coefficient, which represents the ratio of the solid angle, subtended by the central star at the point of observation, to $4 \pi$ (e.g. Gurzadyan 1997)

$W(r)=\frac{1}{2}\left[1-\sqrt{1-\left(\frac{R_{\star}}{r}\right)^{2}}\right]$,

where $R_{\star}$ is the radius of the source of the ionizing photons. Since the luminosity of the hot star per unit frequency interval is given by

$L_{v}=4 \pi R_{\star}^{2} \pi B_{v}\left(T_{\star}\right)$,

we can rewrite Eq. (4) into the form

$N_{\mathrm{H}^{0}}(r, \theta) \int_{v_{0}}^{\infty} \frac{L_{v}}{h v} \frac{W}{\pi R_{\star}^{2}} a_{\nu} \mathrm{e}^{-\tau_{v}} \mathrm{~d} v=N_{\mathrm{p}}(r, \theta) N_{\mathrm{e}}(r, \theta) \alpha_{\mathrm{B}}\left(T_{\mathrm{e}}\right)$.

Denoting the rate of photons from the hot star capable of ionizing hydrogen as

$L_{\mathrm{H}}=\int_{v_{0}}^{\infty} \frac{L_{v}}{h v} \mathrm{~d} v=4 \pi R_{\star}^{2} \int_{v_{0}}^{\infty} \frac{\pi B_{\nu}\left(T_{\star}\right)}{h v} \mathrm{~d} v$

and integrating both sides of Eq. (8) over the radial distance $r$, we get

$\frac{L_{\mathrm{H}}}{2 \pi R_{\star}^{2}}=\int_{R_{\star}}^{r_{\theta}} \frac{N_{\mathrm{p}}(r, \theta) N_{\mathrm{e}}(r, \theta) \alpha_{\mathrm{B}}\left(T_{\mathrm{e}}\right)}{1-\sqrt{1-\left(\frac{R_{\star}}{r}\right)^{2}}} \mathrm{~d} r$,

where $r_{\theta}$ is the radius of the $\mathrm{HI} / \mathrm{H}$ II boundary at the direction $\theta$. To obtain the whole ionization boundary, one needs to solve Eq. (10) for each direction, given by the polar angle $\theta$. Due to the symmetry of the wind with respect to the equatorial plane and considering just the non-crossing streamlines of the gas, it is sufficient to perform calculations from the star's pole $(\theta=0)$ to its equator $(\theta=\pi / 2)$. As in the classical Strömgren sphere calculations, we assume that all atoms of hydrogen with density distribution $N_{\mathrm{H}}(r, \theta)$ are ionized at the ionization radius $r_{\theta}$ (i.e. $N_{\mathrm{p}}=N_{\mathrm{e}} \approx N_{\mathrm{H}}$ ) and neutral outside it (i.e. $N_{\mathrm{p}}=N_{\mathrm{e}} \approx 0$ ). That means that within the ionization boundary we can take $N_{\mathrm{p}} N_{\mathrm{e}}=N_{\mathrm{H}}^{2}$. Nussbaumer \& Vogel (1987) expressed the ionization equilibrium equation in the wind with spherical density distribution in the form

$\frac{L_{\mathrm{H}}}{4 \pi}=\int_{R_{\star}}^{r_{\theta}} N_{\mathrm{p}}(r) N_{\mathrm{e}}(r) \alpha_{\mathrm{B}}\left(T_{\mathrm{e}}\right) r^{2} \mathrm{~d} r$,

which can be derived from Eq. (4) if we adopt the point source approximation (i.e. the dilution factor $\left.W=1 / 4\left(R_{\star} / r\right)^{2}\right)$. This approximation is valid for $R_{\star} / r \ll 1$. However, in our treatment, the ionization boundaries can be close to the central star (especially in the equatorial plane), which requires using Eq. (10) to calculate correctly the $\mathrm{H} \mathrm{I} / \mathrm{H}$ II boundaries, $r_{\theta}$. 
Finally, we express the radial distance $r$ in units of $R_{\mathrm{WD}}$, and define $u$ as

$u \equiv \frac{r}{R_{\mathrm{WD}}}$.

Then, with the further approximation of assuming a constant $T_{\mathrm{e}}$ and thus a constant $\alpha_{\mathrm{B}}$ throughout the ionized nebula, Eq. (10) with the aid of Eq. (1) can be expressed as

$X=\int_{\frac{R_{\star}}{R_{\mathrm{WD}}}}^{u_{\theta}} \frac{\mathrm{d} u}{u^{4}\left(1-\frac{b}{u}\right)^{2 \beta}\left(\frac{\mathrm{d} \mu}{\mathrm{d} \mu_{0}}\right)^{2}\left(1-\sqrt{1-\left(\frac{R_{\star}}{R_{\mathrm{WD}}} \frac{1}{u}\right)^{2}}\right)}$,

where $u_{\theta}=r_{\theta} / R_{\mathrm{WD}}$ and the parameter $X$ is given by

$X=\frac{8 \pi \mu_{\mathrm{m}}^{2} m_{\mathrm{H}}^{2}}{\alpha_{\mathrm{B}}\left(T_{\mathrm{e}}\right)} \frac{R_{\mathrm{WD}}^{3}}{R_{\star}^{2}} L_{\mathrm{H}}\left(\frac{v_{\infty}}{\dot{M}}\right)^{2}$.

The radius of the hot star $R_{\star}$ is determined in terms of its luminosity $L_{\star}$ and temperature $T_{\star}$ as

$R_{\star}=\sqrt{\frac{L_{\star}}{4 \pi \sigma T_{\star}^{4}}}$,

where $\sigma$ is the Stefan-Boltzmann constant. Solutions of Eq. (13) for $u_{\theta}$ at directions $\theta$ define the $\mathrm{H} \mathrm{I} / \mathrm{H} \mathrm{II}$ boundary. First, we introduce some critical parameters of the rotating WD and its wind.

\subsubsection{Rotational velocity of the WD}

Rotational velocities of the accretors in symbiotic binaries are not commonly known. It was observationally found that isolated WDs rotate with $v_{\text {rot }} \sin i \lesssim 40 \mathrm{~km} \mathrm{~s}^{-1}$ (e.g. Heber et al. 1997; Koester et al. 1998). Therefore, it is reasonable to assume that accreting WDs will rotate faster than isolated WDs because of the angular momentum transfer from the accreting material.

Direct evidence for a rapid rotation of accreting WDs is complicated due to a high-density circumstellar medium that shades photospheric absorption lines, which otherwise might be used to derive a possible rotation speed. An exception is the case of the WD in the dwarf nova VW Hyi, for which Sion et al. (1995) derived a rotational velocity of $v_{\text {rot }} \sin i \simeq 600 \mathrm{~km} \mathrm{~s}^{-1}$ by fitting the broad, shallow Si IV absorption doublet in its high-resolution Hubble Space Telescope spectrum. In cases where the energy of the accretor comes solely from the accretion process, the fraction produced by the boundary layer depends on the angular velocity of the accretor. It was found that the observed emission from a boundary layer in cataclysmic variables is significantly lower (around one-quarter) than the flux emitted by the accretion disk, which can be explained by a rapid rotation of the WD accretor (e.g. Hoare \& Drew 1991; Belloni et al. 1991; Vrtilek et al. 1994). Considering the spinning up the accreting star, Popham \& Narayan (1995) derived a theoretical expression for the boundary layer luminosity. Comparing the boundary layer luminosity, inferred from observations, with the theoretical one, Skopal et al. (2005) found that the WD in the symbiotic star EG And rotates at $0.3-0.5$ of its critical velocity. Further support for the rapid rotation of accreting WDs is provided by coherent oscillations in the $U$-light curve of the symbiotic star $\mathrm{CH}$ Cyg with the period of 330-500 s, observed independently during three nights of its quiescent phase (Mikołajewski et al. 1990). The authors interpreted this periodic variability as due to rotation of a magnetic WD in the system.
Accordingly, we first investigated cases with very fast rotational velocities of a WD. However, we found that $v_{\text {rot }} \gtrsim$ $600 \mathrm{~km} \mathrm{~s}^{-1}$ leads to a high compression of the wind, whose particles can cross the equatorial plane for appropriate parameters of the WD wind. This, however, does not satisfy assumptions of the WCZ model. Therefore, to calculate the ionization boundaries within the WCZ model, we examined cases for $v_{\text {rot }} \lesssim 600 \mathrm{~km} \mathrm{~s}^{-1}$.

\subsubsection{Parameters of the hot star wind}

Modelling the broad $\mathrm{H} \alpha$ wings observed in symbiotic binaries by an optically thin bipolar wind from their hot components, Skopal (2006) derived mass loss rates of a few $\times 10^{-8} M_{\odot} \mathrm{yr}^{-1}$ and of a few $\times\left(10^{-7}-10^{-6}\right) M_{\odot} \mathrm{yr}^{-1}$ during quiescent and active phases, respectively. The acceleration parameter $\beta=1.7-1.8$ was in effect for both stages, while the wind terminal velocity during quiescence, $v_{\infty} \sim 1000 \mathrm{~km} \mathrm{~s}^{-1}$ increased to $\sim 2000 \mathrm{~km} \mathrm{~s}^{-1}$, during activity.

For the initial velocity $a$ of the wind in the model (Eq. (3)), we adopted the speed of sound at the interior parts of the ionized wind. According to modelling the extensive wings of emission lines from highly ionized elements by electron scattering, the electron temperature at the vicinity of the hot star, $T_{\mathrm{e}}=$ 30 000-40 000 K (see Fig. 1 of Sekeráš \& Skopal 2012a), corresponds to the speed of sound $a \sim 22 \mathrm{~km} \mathrm{~s}^{-1}$ (monoatomic gas from $\mathrm{H}$ and the adiabatic index of $5 / 3$ were assumed). This value probably represents a lower limit, because $T_{\mathrm{e}}$ within the acceleration zone, i.e. in the vicinity of the burning WD during outbursts $\left(T_{\star} \gtrsim 10^{5} \mathrm{~K}\right)$, can be considerably higher. Furthermore, because of the increase of the hot star luminosity, the terminal velocity of the wind moves from $v_{\infty} \sim 1000$ to $\sim 2000 \mathrm{~km} \mathrm{~s}^{-1}$ during active phases. Thus one can also expect an equivalent increase in the initial velocity of the radiatively driven wind. Accordingly, we adopted $a=20,50$ and $100 \mathrm{~km} \mathrm{~s}^{-1}$ to calculate the $\mathrm{H} \mathrm{I} / \mathrm{H} \mathrm{II}$ boundaries in the wind within the WCZ model.

\subsection{Results}

We calculated the $\mathrm{H} \mathrm{I} / \mathrm{H}$ II boundaries, $u_{\theta}$, according to Eq. (13) for $R_{\star} / R_{\mathrm{WD}}=6$ (Sect. 2.1), $v_{\text {rot }} \lesssim 600 \mathrm{~km} \mathrm{~s}^{-1}$ (Sect. 2.3.1), $v_{\infty}=$ $2000 \mathrm{~km} \mathrm{~s}^{-1}, \beta=1.7$, and $a=20,50,100 \mathrm{~km} \mathrm{~s}^{-1}$ (Sect. 2.3.2) with the aim of finding a range of the parameter $X$, for which a neutral disk-like zone can be created. The results are shown in Fig. 1, which demonstrates that for

$0.002 \lesssim X \lesssim 0.05$

a neutral disk-like zone can be created at the equator of the hot star, flaring away from it. For $X \lesssim 0.002$, the ionization boundary tends to enclose the star, while $X \gtrsim 0.002$ moves the boundary to farther distances with its simultaneous narrowing, until it disappears for $X \gtrsim 0.05$. For a given set of parameters, different compression of the outflowing material is caused by a different WD rotation. Higher rotational velocities lead to a higher compression of the wind towards the equatorial plane and to wider range of the parameter $X$, resulting in creation of the neutral disk-like zone (see Fig. 1).

Shaping of the ionization boundaries is also sensitive to the wind parameters $a, v_{\infty}$, and $\beta$. For a higher initial velocity $a$ or terminal velocity $v_{\infty}$, the corresponding ionization boundary lies farther from the hot star. This is because higher velocities lead to lower densities in the wind, which moves the point of the balance between the flux of ionizing photons and neutral atoms to farther 
Z. Cariková and A. Skopal: Ionization structure of hot components in symbiotic binaries during active phases
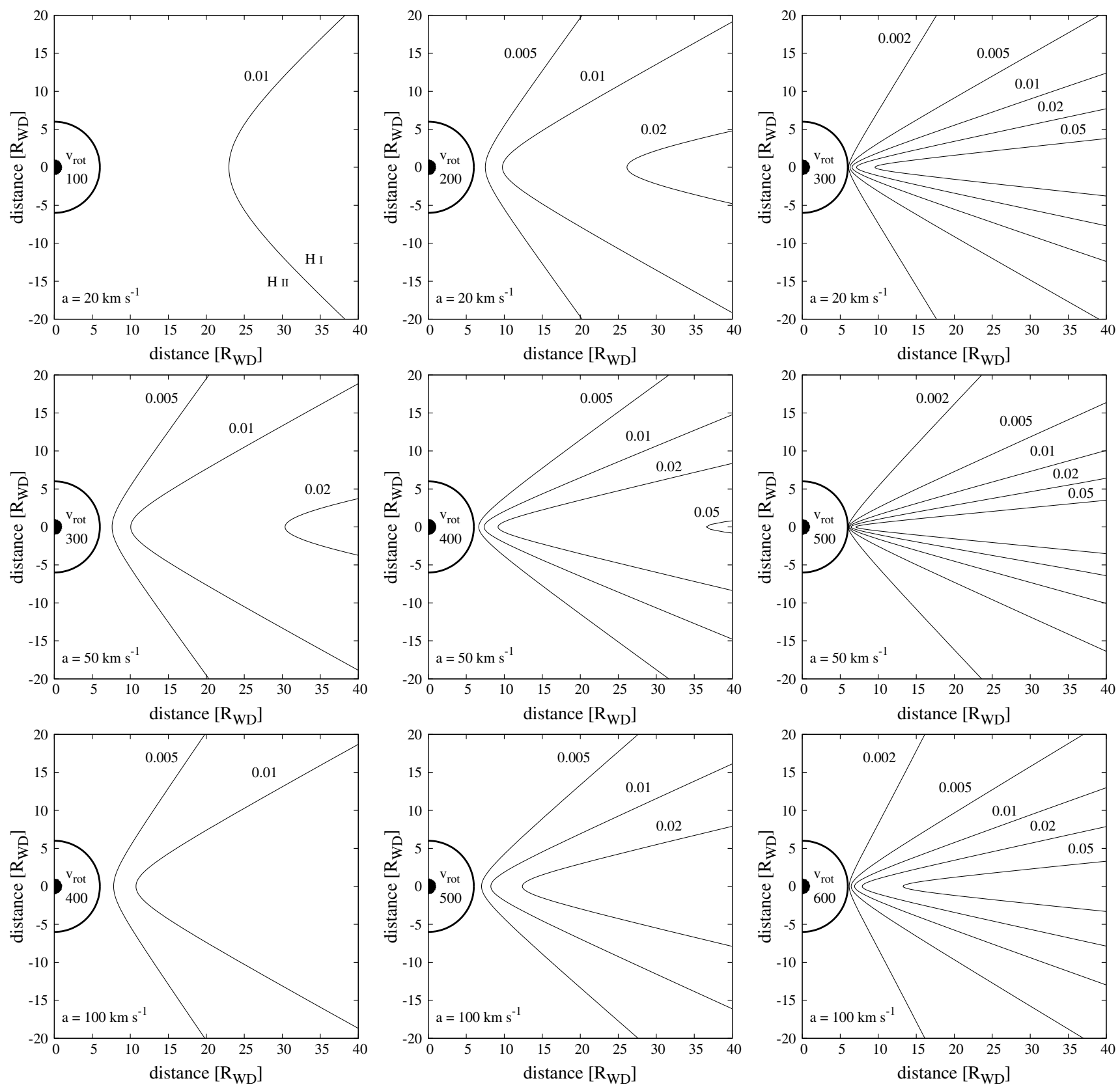

Fig. 1. H I/H II boundaries in the wind compression model (Sect. 2) at the vicinity of the hot star (solid thick circle). They were calculated according to Eq. (13) for $a=20,50$, and $100 \mathrm{~km} \mathrm{~s}^{-1}$, displayed at the top, middle, and bottom row of panels, respectively, with fixed $v_{\infty}=2000 \mathrm{~km} \mathrm{~s}{ }^{-1}$ and $\beta=1.7$. The rotational velocities, $v_{\text {rot }}$, are in $\mathrm{km} \mathrm{s}^{-1}$. The boundaries are labelled by the corresponding parameter $X$. The neutral H I zone extends from the boundary in directions outside the star. The remainder part of the wind is ionized. Boundaries are axially symmetric with respect to the spin axis of the WD (the black circle), assumed to be perpendicular to the orbital plane. Distances are in units of the WD radius.

distances. On the other hand, a higher value of the parameter $\beta$ accelerates the wind from $a$ to $v_{\infty}$ more slowly, leading to higher densities in the wind and thus to a closer position of the neutral zone to the hot star, which is characterized by a larger opening angle.

The range of the ionization parameter $X$, for which the neutral disk-like zone can be created, is important for comparison with observations.

\section{Comparison with observations}

Application of the wind compression model to the hot components of symbiotic binaries during active phases revealed the possibility of forming a neutral disk-like zone around the hot star. This confirmed theoretically the previous suggestion based on modelling the UV/near-IR SED that there is a neutral flared disk surrounding the active hot star at the orbital plane (see Fig. 27 of Skopal 2005). The model is also consistent with the ionization structure derived from the photometric and spectroscopic analysis of the eclipsing symbiotic binary AX Per during its recent active phase (see Fig. 9 of Skopal et al. 2011).

In the following sections, we verify the model by comparing its basic properties with those obtained independently from observations. In Sect. 3.1, we investigate the dependence of the wind compression, given by the parameter $X$, on the mass loss rate from the hot star. The aim is to determine if the presence or absence of the neutral disk around the hot stars during active or quiescent phases is consistent with the model predictions. In 
Sect. 3.2, we compare the model column density of the neutral hydrogen, calculated throughout the neutral zone, with that derived from observations. Finally, in Sect. 3.3, we compare the observed emission measure to that given by the model.

\subsection{Wind compression as a function of $\dot{M}$}

According to Eq. (13), the ionization structure in WCZ model is given by the parameter $X$. It reflects a degree of compression of the wind (Fig. 1). The changes of the $X$ parameter are dominated by significant changes in the mass loss rate from the hot star during different phases of the star's activity (Sect. 2.3.2). Therefore, to probe if the presence or absence of the neutral disk in a symbiotic system is consistent with the WCZ model, we investigate a dependence of $X$ on $\dot{M}$ for physical parameters of selected symbiotic stars during quiescent and active phases. According to Eq. (14), the value of the parameter $X$ is given by the hot star parameters $L_{\mathrm{H}}, R_{\star}$ and those of its wind, $\dot{M}, v_{\infty}$.

\subsection{1. $X(\dot{M})$ relationship during quiescent phases}

For a quiescent phase, we selected ten objects with available $L_{\mathrm{h}}$, $R_{\mathrm{h}}^{\text {eff }}, T_{\mathrm{h}}$ of their hot components, as derived by modelling the UV/near-IR SEDs (EG And, Z And, CI Cyg, LT Del, YY Her, RW Hya, SY Mus, AG Peg and AX Per; see Table 3 of Skopal 2005) and X-ray/near-IR SED (AG Dra; see Table 3 of Skopal et al. 2009). $L_{\mathrm{H}}$ is determined by $L_{\mathrm{h}}$ and $T_{\mathrm{h}}$. Parameters of the wind were taken from Table 1 of Skopal (2006). For AG Dra and LT Del, we adopted average values $v_{\infty} \equiv 1000 \mathrm{~km} \mathrm{~s}^{-1}$ and $\dot{M} \equiv 5 \times 10^{-8} M_{\odot} \mathrm{yr}^{-1}$. In the case of EG And, we used $v_{\infty}=$ $900 \mathrm{~km} \mathrm{~s}^{-1}$ and $\dot{M}=5 \times 10^{-9} M_{\odot} \mathrm{yr}^{-1}$, according to Mürset et al. (1997).

To get a rough estimate of the $X(\dot{M})$ quantities, we first used fundamental parameters $L_{\mathrm{h}}, R_{\mathrm{h}}^{\text {eff }}, T_{\mathrm{h}}$ as derived from observations. The results are plotted in Fig. 2 by full grey circles. The "plus" sign corresponds to AG Dra, which is seen more from the pole, and thus its $R_{\mathrm{h}}^{\text {eff }} \approx R_{\star}$ (see Sect. 2.1). This implies that the other nine systems (all with a high orbital inclination) have $R_{\mathrm{h}}^{\text {eff }}>R_{\star}$, which corresponds to a lower value of $X$ (see Eq. (14)). To improve the results, we determined the $X(\dot{M})$ quantities for the hot star parameters $L_{\star}, R_{\star}, T_{\star}$ as follows. (i) We put $T_{\star} \equiv T_{\mathrm{h}}=160000 \mathrm{~K}$, as derived by modelling the $\mathrm{X}$-ray/UV SED of AG Dra. Such a high temperature is also supported by a direct X-ray observation of a sample of symbiotic stars with a pronounced supersoft $\mathrm{X}$-ray $(0.1-0.4 \mathrm{keV})$ radiation that is similar in the profile with that of AG Dra (see Fig. 2 of Mürset et al. 1997). (ii) We determined $R_{\star}$ and $L_{\mathrm{H}}$ for $L_{\star}=L_{\mathrm{h}}$. Corresponding $X(\dot{M})$ values are plotted in Fig. 2 by open circles.

According to Eq. (14), the $X(\dot{M})$ relationship can be written in the form

$\log (X)=C-2 \log (\dot{M})$,

where $C=\log \left(9.6 \times 10^{-34} R_{\mathrm{WD}}^{3} / R_{\star}^{2} L_{\mathrm{H}}\right)+2 \log \left(v_{\infty}\right)$ for $\alpha_{\mathrm{B}}(\mathrm{H}, 20000 \mathrm{~K})=1.43 \times 10^{-13} \mathrm{~cm}^{3} \mathrm{~s}^{-1}$ (e.g. Hummer \& Seaton $1963)$ and given parameters. Fits to filled circles and to open circles with the "plus" sign in Fig. 2 correspond to $C=-14.81$ and -14.26 , respectively (for $\dot{M}$ in $M_{\odot} \mathrm{yr}^{-1}$ ). This shows that the quantities of $X$ for the hot star parameters $\left(L_{\star}, R_{\star}, T_{\star}\right)$ are a factor of $\sim 3.5$ higher than those determined using the observed parameters $\left(L_{\mathrm{h}}, R_{\mathrm{h}}^{\text {eff }}, T_{\mathrm{h}}\right)$. Thus, regardless of the selected parameters, both sets of the $X(\dot{M})$ data are located well above the grey belt in the figure, i.e. without the possibility of creation of the flared neutral disk-like zone, as predicted by the WCZ model

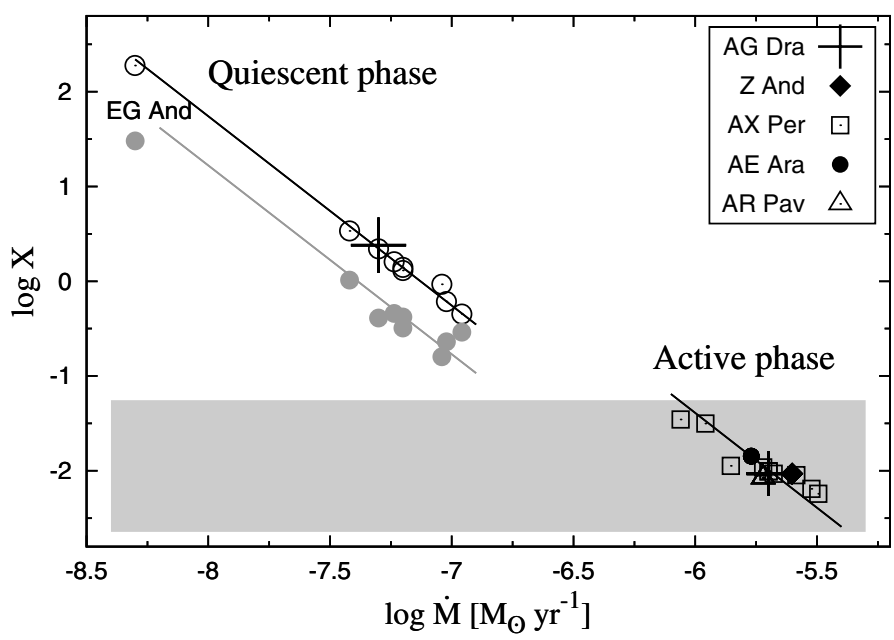

Fig. 2. Parameter $X$ as a function of $\dot{M}$ from the hot star, as given by physical parameters of the selected symbiotic stars during quiescent (left) and active (right, keys) phases. Their fits with Eq. (17) are denoted by the solid lines. The grey belt corresponds to $X=0.002-0.05$, which leads to creation of the neutral zone around the hot star in the form of a flared disk (see Fig. 1, Sects. 2.3 and 2.4).

(Sect. 2.4, relation (16)). In other words, the mass loss rate from the hot star during quiescent phases is too small to give rise to a neutral zone of hydrogen, because the rate of ionizing photons is sufficiently high to ionize even the compressed fraction of the wind.

\subsection{2. $X(\dot{M})$ relationship during active phases}

Determination of the hot star parameters during active phases for systems with a high orbital inclination is complicated by the presence of the edge-on disk, which blocks the original radiation from the hot star in the direction of the observer (see Sect. 5.3.6 of Skopal 2005). Therefore, indirect methods based on the ratio of nebular line fluxes and/or the presence of the highest ionization states in the spectrum (Mürset \& Nussbaumer 1994) are employed to estimate the hot star temperature during active phases. For example, using the ratio of He II $4686 \AA$ and $\mathrm{H} \beta$ line fluxes from the spectra of the symbiotic prototype $\mathrm{Z}$ And during its 2000-03 outburst, Sokoloski et al. (2006) derived $T_{\mathrm{h}}=92000 \pm 20000$ to $160000 \pm 35000 \mathrm{~K}$. During the smaller, 1997 outburst, both methods indicated an increase in $T_{\mathrm{h}}$ to $180000 \mathrm{~K}$.

More trustworthy estimates of $T_{\mathrm{h}}$ can be achieved only for non-eclipsing systems with the aid of multiwavelength modelling of the supersoft X-ray/UV SED, which covers the total spectrum emitted by the burning WD. An applicable system here is AG Dra, the strongest supersoft source among classical symbiotic stars. Although the supersoft component disappears during its active phases, the very high far-UV fluxes and a strong nebular continuum constrain $T_{\mathrm{h}}$ to be $>150-180 \mathrm{kK}$, depending on the outburst stage (see Skopal 2005; Skopal et al. 2009). As the orbital inclination of AG Dra is low $\left(i \approx 30-60^{\circ}\right.$, Mikołajewska et al. 1995; Schmid \& Schild 1997), we can directly see the WD's pseudophotosphere and thus can assume that its $T_{\star} \approx T_{\mathrm{h}}$ and $R_{\star} \approx R_{\mathrm{h}}^{\text {eff }}$ (see also Sect. 2.1). Therefore, we adopted the average hot star parameters, which were derived from the model SEDs of AG Dra, $T_{\star}=170000 \pm 7000 \mathrm{~K}$, and $R_{\star}=0.06 \pm 0.01 R_{\odot}$, (i.e. $L_{\star}=2700 \pm 700 L_{\odot}$ and $L_{\mathrm{H}}=$ $\left.(1.4 \pm 0.4) \times 10^{47} \mathrm{~s}^{-1}\right)$, as representative values for the active 
phases of AG Dra, Z And, AX Per, AE Ara, and AR Pav. The parameters $v_{\infty}$ and $\dot{M}$ for these systems are available in Table 1 of Skopal (2006) and Table 7 of Skopal et al. (2011). The $X(\dot{M})$ quantities of active symbiotic stars lie well within the possibility of creation of the flared neutral disk-like zone predicted by the WCZ model (the grey belt in Fig. 2). In other words, during active phases the mass loss rate is so high that the rate of ionizing photons is not capable of ionizing the compressed wind around the hot star equator.

Figure 2 suggests that the presence or absence of the neutral disk-like zone in a symbiotic system during active or quiescent phases, respectively, is given by the value of the parameter $X$. During quiescent phases $X \approx 1$, while during active phases $X \approx 0.01$. This large difference results from the very different values of $\dot{M}$ in quiescent and active phases (Sect. 2.3.2) and the proportionality of $X$ to $\dot{M}^{-2}$. Thus, we can conclude that creation of the neutral disk-like structure, indicated in the spectrum of active symbiotic binaries, is consistent with the WCZ model, i.e. it can be a result of a compression of the hot star wind, which is significantly enhanced during active phases.

\subsection{Column density of the neutral hydrogen}

In this section, we verify the model by comparing the column density of the neutral hydrogen atoms, which was calculated throughout the neutral disk-like zone, with that derived from observations during active phases of symbiotic binaries.

Modelling the SED of symbiotic binaries during active phases showed the presence of a warm $\left(1-2 \times 10^{4} \mathrm{~K}\right)$ disk-like pseudophotosphere (Sect. 1) and a large amount of the neutral hydrogen near to the orbital plane of the binary. Column densities derived from the ultraviolet spectra are between $\sim 10^{21}$ and a few $\times 10^{23} \mathrm{~cm}^{-2}$ (Skopal 2005). A large amount of the neutral material can be identified even around the orbital phase $\sim 0.5$, when the hot component is in front of the cool giant. Figure 3 demonstrates this case for BF Cyg. During the active phase, the radiation of the warm pseudophotosphere was Rayleigh attenuated with $n_{\mathrm{H}}=4.8 \times 10^{22} \mathrm{~cm}^{-2}$ (the grey part in the figure), while during quiescence, the hot component radiates at $T_{\mathrm{h}}>10^{5} \mathrm{~K}$ with no signatures of the Rayleigh scattering and the iron curtain absorptions. This demonstrates that the presence/absence of the large amount of the neutral hydrogen on the line of sight is connected with intrinsic variations of the circumstellar matter around the hot star during active/quiescent phases. A contribution from the interstellar matter (ISM) in the direction to BF Cyg can be neglected. For $E_{B-V}=0.35$ mag (Mürset et al. $1991), n_{\mathrm{H}}(\mathrm{ISM}) \sim 1.7 \times 10^{21} \mathrm{~cm}^{-2}$ (e.g. Diplas \& Savage 1994), which is difficult to measure on the low-resolution International Ultraviolet Explorer spectra.

In the WCZ model, we calculated the column density of the neutral hydrogen atoms in the radial direction $\theta$ throughout the neutral zone as a function of $r$,

$n_{\mathrm{H}}\left(\theta, r \geq r_{\theta}\right)=\int_{\mathrm{r} \geq \mathrm{r}_{\theta}}^{\infty} N_{\mathrm{H}}(r, \theta) \mathrm{d} r$

where the density distribution in the wind, $N_{\mathrm{H}}(r, \theta)$, is given by Eq. (1). Figure 4 shows two $n_{\mathrm{H}}\left(\theta, r \geq r_{\theta}\right)$ functions corresponding to ionization boundaries calculated for $X=0.01$, $v_{\text {rot }}=300 \mathrm{~km} \mathrm{~s}^{-1}, a=20 \mathrm{~km} \mathrm{~s}^{-1}$ (see the top right panel in Fig. 1), and two different orbital inclinations $i=\theta=80^{\circ}$ and $90^{\circ}$. The edge-on view $\left(\theta=90^{\circ}\right)$ gives a maximum value of the column density, since we are looking through the densest parts of the compressed wind. Calculations were performed for
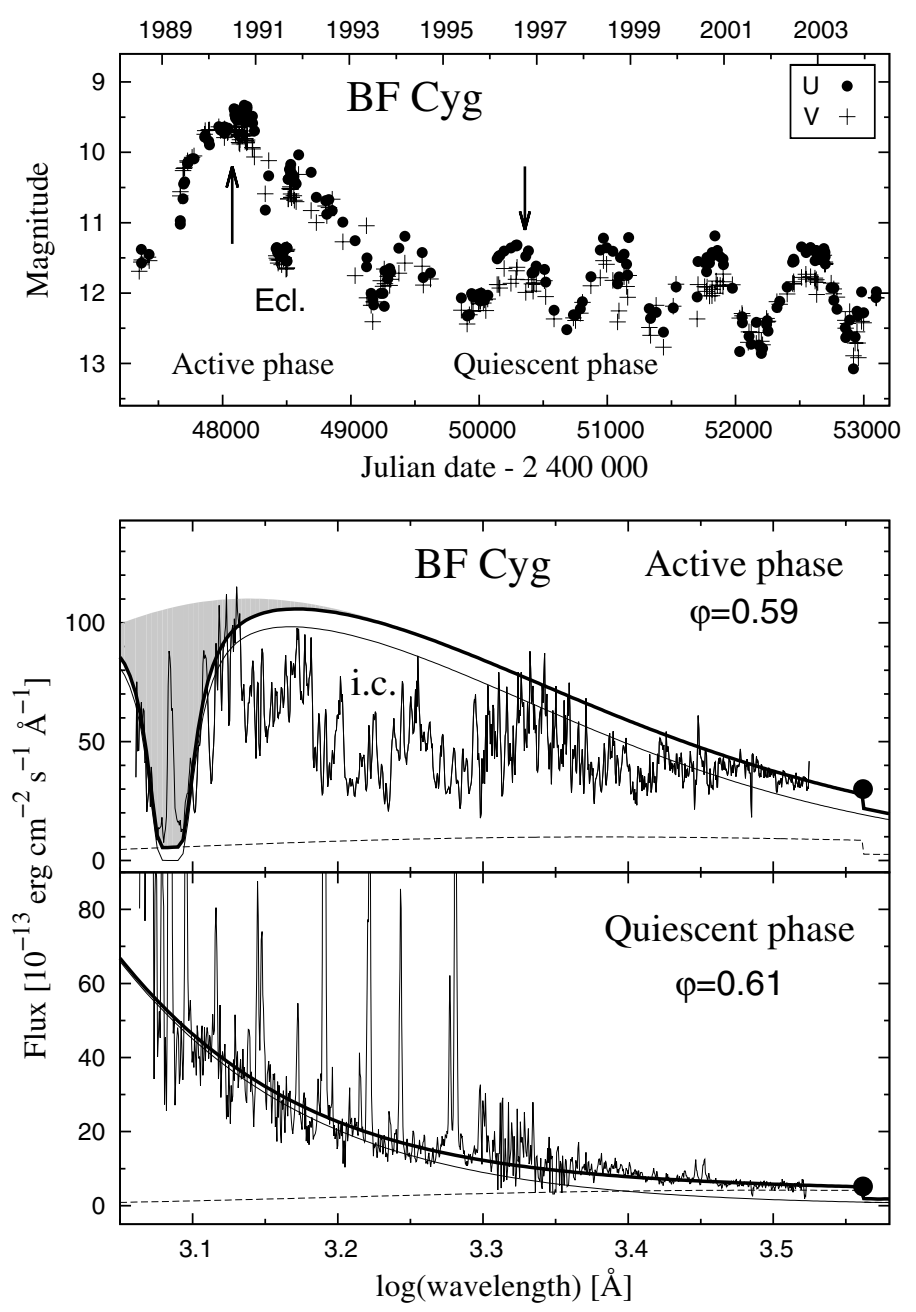

Fig. 3. Ultraviolet SED of BF Cyg during its 1990 optical maximum (mid) and the following quiescent phase (bottom). Times of observations are denoted by arrows at the top panel with the light curves. In both cases, the binary was seen from the same position with the hot star in front (orbital phase $\varphi \sim 0.6$ ). The dashed and the thin solid line represent the continuum from the nebula and the hot star, respectively. The thick solid line is the model SED. The grey surface marks the Rayleigh attenuated continuum with $n_{\mathrm{H}}=4.8 \times 10^{22} \mathrm{~cm}^{-2}$, and strong blended absorptions of the iron curtain are denoted by "i.c.".

$T_{\star}=1.7 \times 10^{5} \mathrm{~K}$ and $L_{\star}=3 \times 10^{3} L_{\odot}$, i.e. $L_{\mathrm{H}}=1.6 \times 10^{47} \mathrm{~s}^{-1}$ (Sect. 3.1.2), and the wind parameters, $\dot{M}=2 \times 10^{-6} M_{\odot} \mathrm{yr}^{-1}$, $v_{\infty}=2000 \mathrm{~km} \mathrm{~s}^{-1}$, which represent mean values measured during active phases (see Table 1 of Skopal 2006).

To compare the observed values of $n_{\mathrm{H}}$ with the model (i.e. $n_{\mathrm{H}}\left(\theta, r=R_{\mathrm{d}}\right)$, see Eq. (18)), we need to know the radius of the optically thick disk, $R_{\mathrm{d}}$, whose outer rim represents the warm pseudophotosphere indicated during active phases of symbiotic binaries with a high orbital inclination (Sect. 5.3.5 and Fig. 27 in Skopal 2005). Therefore, a rough estimate of $R_{\mathrm{d}}$ can be obtained from the effective radius, $R_{\mathrm{h}}^{\text {eff }}$, of the hot components derived from the SED. According to its definition (here in Sect. 2.1), we can write

$4 \pi\left(R_{\mathrm{h}}^{\mathrm{eff}}\right)^{2} \sigma T_{\mathrm{h}}^{4}=2 \pi R_{\mathrm{d}} 2 H \sigma T_{\mathrm{h}}^{4}$,

where $H$ is the height of the flared disk (see also Fig. 6). For a canonical value of $H / R_{\mathrm{d}} \equiv 0.3$ during active phases 
Table 1. Column densities $n_{\mathrm{H}}$, effective radii of the hot components $R_{\mathrm{h}}^{\text {eff }}$, disk radii $R_{\mathrm{d}}$, and emission measures $E M$ derived from observations.

\begin{tabular}{lccccc}
\hline \hline Object & $\begin{array}{c}\log n_{\mathrm{H}} \\
\left(\mathrm{cm}^{-2}\right)\end{array}$ & $\begin{array}{c}R_{\mathrm{h}}^{\text {eff }} \\
\left(R_{\odot}\right)\end{array}$ & $\begin{array}{c}R_{\mathrm{d}}^{1)} \\
\left(R_{\odot}\right)\end{array}$ & $\begin{array}{c}R_{\mathrm{d}}^{2)} \\
\left(R_{\odot}\right)\end{array}$ & $\begin{array}{c}E M \\
\left(10^{59} \mathrm{~cm}^{-3}\right)\end{array}$ \\
\hline BF Cyg & $22.68_{-0.30}^{+0.18}$ & 7.1 & 13.0 & $24 \pm 8.3$ & $<120$ \\
CI Cyg & $23.00_{-0.30}^{+0.18}$ & 0.67 & 1.23 & $22 \pm 8.0$ & 5.3 \\
YY Her & $23.14_{-0.30}^{+0.18}$ & 0.89 & 1.63 & $20 \pm 8.0$ & 7.2 \\
AR Pav & $22.65_{-0.30}^{+0.18}$ & 1.90 & 3.48 & $21 \pm 8.0$ & 17 \\
AX Per & $22.84_{-0.30}^{+0.18}$ & 0.42 & 0.77 & - & 3.6 \\
& - & 6.2 & 11.3 & - & 3.5 \\
& - & 8.2 & 15.0 & - & 3.8 \\
& - & 11 & 20.1 & $22 \pm 2.0$ & 8.2 \\
AG Dra & - & 7.0 & 12.8 & - & 4.0 \\
\hline
\end{tabular}

Notes. Data are from Skopal (2005) and Skopal et al. (2011). 1) According to Eq. (19), ${ }^{2)}$ from the eclipse profile (see text).

(Skopal 2006), $R_{\mathrm{d}} \sim 2 \times R_{\mathrm{h}}^{\text {eff. }}$ Table 1 summarizes values of $R_{\mathrm{h}}^{\text {eff }}$ from Table 4 of Skopal (2005) and Table 8 of Skopal et al. (2011).

The disk radius can also be estimated directly from eclipses, which develop in optical light curves during active phases (e.g. Belyakina 1991; Munari 1992; Brandi et al. 2005; Skopal 2008, Fig. 4 here). For the 1991 eclipse of BF Cyg, the radius of the eclipsed object in units of the binary components separation, $A$, was $R_{\mathrm{e}} / A=0.051 \pm 0.018$, which yields $R_{\mathrm{e}}\left(\equiv R_{\mathrm{d}}\right)=24 \pm 8.3 R_{\odot}$ for the total mass of the binary $M_{\mathrm{T}}=2.5 M_{\odot}$ and the orbital period of 757.3 days (Skopal et al. 1997, and references therein). CI Cyg: the first two contact times of its 1975 eclipse, $T_{1}=\mathrm{JD} 2442634.5 \pm 1.5, T_{2}=\mathrm{JD} 2442646.8 \pm 2.0$ (from the light curve of Belyakina 1991) and its middle, $\operatorname{JD}_{\mathrm{Ecl} .}(1975.8)=$ $2442691.6 \pm 0.8$ (Skopal 1998), orbital period of 853.8 days (Fekel et al. 2000) and $M_{\mathrm{T}}=2.0 M_{\odot}$ (Kenyon et al. 1991) give $R_{\mathrm{d}}=22 \pm 8 R_{\odot}$. YY Her: the contact times of the eclipse that developed during the 1993-94 active phase (Skopal 2005), the orbital period of 574.6 days (Wiecek et al. 2010) for $M_{\mathrm{T}} \equiv 2.0 M_{\odot}$ gives $A=367 R_{\odot}$ and thus $R_{\mathrm{d}}=20 \pm 8 R_{\odot}$. AR Pav: during epochs 59-63 (1987-1995) of its historical light curve, Skopal et al. (2000) estimated $R_{\mathrm{e}} / A=0.05 \pm 0.02$ (see Fig. 1 there), which for $A=419 R_{\odot}$ (Schild et al. 2001) implies $R_{\mathrm{d}}=21 \pm 8 R_{\odot}$. AX Per: recently, Skopal et al. (2011) derived $R_{\mathrm{d}}=27 \pm 2 R_{\odot}$ from the 2009 eclipse, which developed during the 2007-10 active phase. The eclipse profile was asymmetric, with the first two contact times corresponding to $R_{\mathrm{d}}=22.3 \pm 2 R_{\odot}$, in particular agreement with the disk radius derived from the model SED $\left(R_{\mathrm{h}}^{\text {eff }}=11 R_{\odot}\right.$ and Eq. (19)). It is important to note that the radius of the warm disk-like shell, derived from the eclipse profile, represents an upper limit because of a measurable contribution of the ionized part of the hot star wind at radial distances of $\sim 20 R_{\odot}$ $\left(E M\left(r>20 R_{\odot}\right) \sim 10^{57} \mathrm{~cm}^{-3}\right.$ in the model). This also causes large uncertainties of $R_{\mathrm{d}}$ from eclipses.

Figure 4 compares quantities of $n_{\mathrm{H}}$ derived from observations of symbiotic stars with a high orbital inclination, BF Cyg, CI Cyg, YY Her, AR Pav, and AX Per. We used column densities derived from the Rayleigh scattering of the far-UV continuum photons around the Ly- $\alpha$ line on the neutral atoms of hydrogen. The errors in $n_{\mathrm{H}}$ this way derived are of the order of $50 \%$ because of the influence of other absorption effects (Dumm et al. 1999). For the purpose of this work, we used values of $n_{\mathrm{H}}$ for the

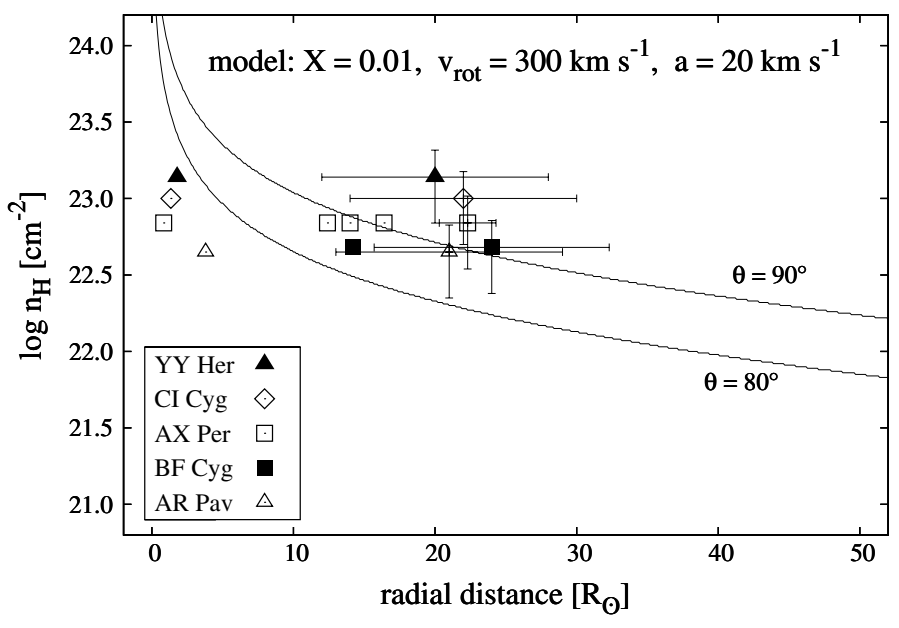

Fig. 4. Comparison of the model hydrogen column densities $n_{\mathrm{H}}\left(90^{\circ}, r \geq\right.$ $\left.r_{\theta}\right)$ and $n_{\mathrm{H}}\left(80^{\circ}, r \geq r_{\theta}\right)$ (solid lines), calculated according to Eq. (18), with those derived from observations of selected eclipsing objects, plotted at the distance $r=R_{\mathrm{d}}$ from the WD centre (Table 1).

above-mentioned symbiotic stars during their outbursts at the orbital phases between 0.3 and 0.6 to eliminate a larger influence of the neutral wind from the giant (Table 1). Nevertheless, the measured values of $n_{\mathrm{H}}$ can still contain a contribution from the giant's wind, which is in the neutral form at/around the orbital plane during active phases, because the ionizing photons are blocked by the neutral disk-like formation surrounding the hot star. For the mass loss rate from the giant of a few $\times 10^{-7} M_{\odot} \mathrm{yr}^{-1}$ (Skopal 2005), the column density of its wind in the direction to the hot component at the orbital phase 0.5 and $i=90^{\circ}$ is of $\approx 1 \times 10^{22} \mathrm{~cm}^{-2}$ (Sekeráš \& Skopal 2012b).

In spite of large errors in $n_{\mathrm{H}}$ and $R_{\mathrm{d}}$, often derived from nonsimultaneous observations, Fig. 4 shows that the $n_{\mathrm{H}}$ values are consistent with those calculated within the WCZ model for parameters of the hot star and its wind derived from observations during active phases.

\subsection{Emission measure of the ionized wind from the hot star}

In this section we calculate the emission measure, $E M$, of the ionized part of the hot star wind from regions above/below the neutral disk-like zone and compare it to that derived from observations during active phases.

Modelling the SED of symbiotic binaries during active phases showed that the emission measure of the nebular component of radiation is between a few $\times 10^{58}$ and a few $\times 10^{60} \mathrm{~cm}^{-3}$ (see Table 4 of Skopal 2005). In addition, the modelling revealed the presence of a low-temperature nebula (LTN, $T_{\mathrm{e}} \sim 14000 \mathrm{~K}$ ), which is subject to eclipses, and a high-temperature nebula $\left(\mathrm{HTN}, T_{\mathrm{e}}>30000 \mathrm{~K}\right.$ ), which is seen during eclipses as the only component. Later, Skopal (2006) ascribed the LTN to the ionized wind from the hot star, which develops during active phases. In our model, $E M$ is determined as

$E M=\int_{V} N_{\mathrm{H}}^{2}(r, \theta) \mathrm{d} V$,

where $N_{\mathrm{H}}(r, \theta)$ is the density of the ionized hydrogen given by Eq. (1), calculated throughout the volume $V$ of the H II zone. In the spherical coordinates $(r, \theta, \phi)$, the volume element $\mathrm{d} V=$ $r^{2} \sin \theta \mathrm{d} r \mathrm{~d} \theta \mathrm{d} \phi$, and in the WCZ model, the density distribution is azimuthally symmetric (i.e. along the coordinate $\phi$ ). This 
Z. Cariková and A. Skopal: Ionization structure of hot components in symbiotic binaries during active phases

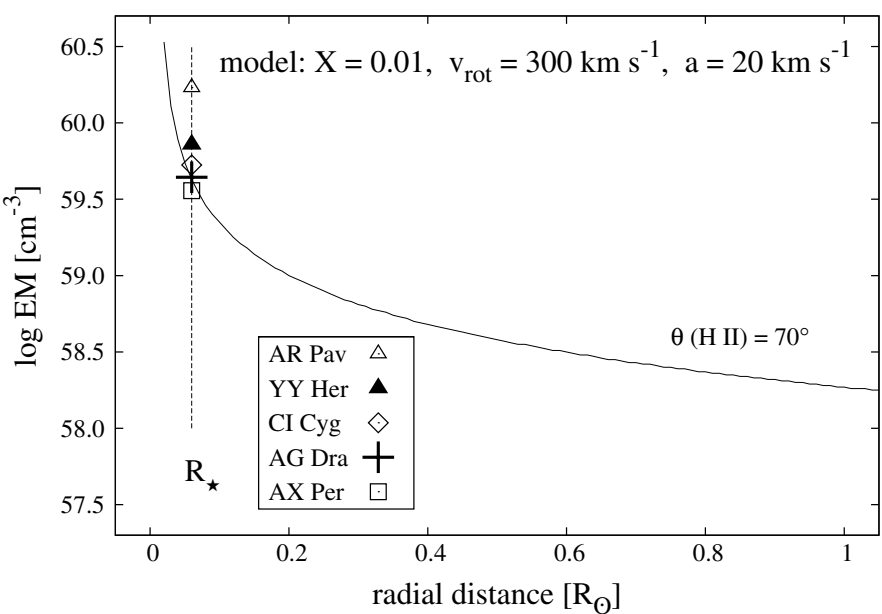

Fig. 5. Comparison of the model emission measure calculated according to Eq. (21) throughout the $\mathrm{H}$ II region as a function of the radial distance $r$ (solid line) with the values derived from the spectra of selected symbiotic stars during active phases (Table 1$). \theta(\mathrm{H}$ II) is the opening angle of the H II zone. The same model as in Fig. 4 was selected.

allows us to express Eq. (20) as

$E M=4 \pi \int_{R_{\star}}^{\infty} \int_{0}^{\theta(\mathrm{H} \mathrm{II})} N_{\mathrm{H}}^{2}(r, \theta) r^{2} \sin \theta \mathrm{d} r \mathrm{~d} \theta$,

where $\theta(\mathrm{HII})$ is the $\theta$ coordinate of the $\mathrm{HI} / \mathrm{H}$ II boundary at the given $r$. Figure 5 shows the calculated $E M$ of the ionized part of the wind as a function of $r$ for the same model that we used to calculate $n_{\mathrm{H}}$ in Sect. 3.2. It shows that the observed values of the emission measure, as derived by modelling the SED (i.e. $E M_{\text {LTN }}$ in Table 4 of Skopal 2005, Table 1 here), are in a good agreement with the model prediction. We excluded the case of BF Cyg, because it was not possible to extract the LTN component from the SED. A problem in comparing the observed and modelled emission measure is connected with a shielding effect for objects with a high orbital inclination. In the real case, it is probable that the outer rim of the flared disk will shield the inner parts of the ionized wind. As a result, the calculated emission measure $E M_{\mathrm{cal}}>E M_{\mathrm{LTN}}$. However, we did not include the shielding effect in the calculation, because the orbital inclination and the opening angle of the neutral zone are not well-known parameters.

According to Fig. 5, we can conclude that the emission measure of the ionized wind from the hot star, calculated within the WCZ model (Eq. (21)), is consistent with that derived from observations by modelling the SED of symbiotic binaries during active phases.

\section{Conclusion}

We investigated the ionization structure around the hot stars in symbiotic binaries during active phases. Applying the wind compression model (Sect. 2), we found that a neutral disk-like zone can be formed as a result of the enhanced wind from the rotating WD at its equatorial plane. Around the pole directions, above/below the neutral disk, the wind is diluted by a fast rotation, which makes it easily ionized by the central hot star (i.e. the WD's pseudophosphere in the model). Examples of the corresponding $\mathrm{HI} / \mathrm{H}$ II boundaries are shown in Fig. 1. A representative model of the ionization structure of the hot component in a symbiotic binary during active phase is shown in Fig. 6.

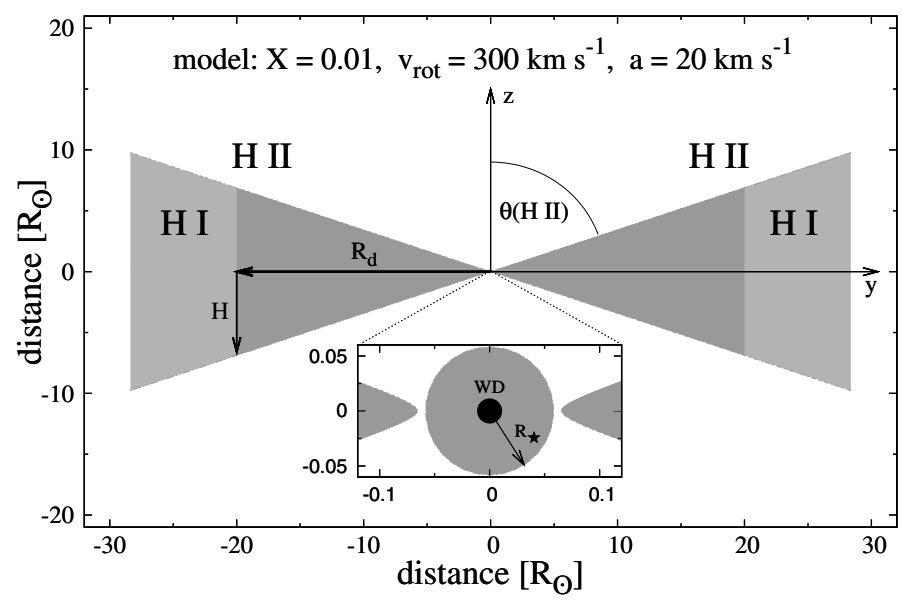

Fig. 6. Model of the ionization structure of the hot components in symbiotic binaries during active phases (Sect. 2, Eq. (13)) as seen on a cut perpendicular to the orbital $(x, y)$ plane containing the WD, which spins along the $z$-axis. The neutral zone has the form of a flared disk. It is optically thick to the distance $R_{\mathrm{d}}$, i.e. the radius of the warm pseudophotosphere. The ionized zone has the opening angle $\theta(\mathrm{HII})$. The model corresponds to $R_{\mathrm{h}}^{\text {eff }} \sim 11 R_{\odot}$ (see Eq. (19)). The inplot figures the central WD as a black circle with the radius $R_{\mathrm{WD}}$, which is the source of the optically thick wind. The wind becomes optically thin at the distance $R_{\star}$ (the shadow circle), which simulates the WD's pseudophotosphere, the source of ionizing photons.

Applying the wind compression model to physical parameters of the hot stars in symbiotic binaries, we found that the neutral disk-like zone can be created during active phases, when the mass loss rate enhances to $\sim 2 \times 10^{-6} M_{\odot} \mathrm{yr}^{-1}$. However, during quiescent phases, the formation of the neutral region is unlikely because of insufficient mass loss rate only of a few $\times 10^{-8} M_{\odot} \mathrm{yr}^{-1}$ (Sect. 3.1, Fig. 2).

We verified the model by comparing the hydrogen column density, calculated in the radial direction from the hot star throughout the Hi zone and the emission measure of the H II zone, with quantities derived from observations (Sects. 3.2 and 3.3). Figures 4 and 5 show that both parameters are consistent with those calculated according to the wind compression model for $\dot{M}=2 \times 10^{-6} M_{\odot} \mathrm{yr}^{-1}$ and $v_{\infty}=2000 \mathrm{~km} \mathrm{~s}^{-1}$, which represent mean values derived from observations during active phases.

In view of these results, we propose that the flared optically thick disks, indicated in the spectrum of active symbiotic binaries with a high orbital inclination, can form from the enhanced wind, which is compressed towards the equatorial plane due to a fast rotation of its source, the central WD. In this way we justified the ionization structure of the hot components in symbiotic binaries during active phases, as we had suggested in previous papers (e.g. Skopal 2005, 2006; Skopal et al. 2006, 2011).

Acknowledgements. The authors thank the anonymous referee for constructive comments. This research was supported by a grant of the Slovak Academy of Sciences, VEGA No. 2/0038/10 and by the Project ITMS No. 26220120029, based on the supporting operational Research and development program financed from the European Regional Development Fund.

\section{References}

Allen, D. 1980, MNRAS, 190, 75

Belloni, T., Verbunt, F., Beuermann, K., et al. 1991, A\&A, 246, L44 Belyakina, T. S. 1991, Izv. Krymsk. Astrofiz. Obs., 83, 118 
Bisikalo, D. V., Boyarchuk, A. A., Kilpio, E. Yu., Tomov, N. A., \& Tomova, M. T. 2006, Astron. Rep., 80, 722

Bjorkman, J. E., \& Cassinelli, J. P. 1993, ApJ, 409, 429

Boyarchuk, A. A. 1967, SvA, 11, 8

Brandi, E., Mikolajewska, J., Quiroga, C., et al. 2005, A\&A, 440, 239

Crocker, M. M., Davis R. J., Spencer, R. E., et al. 2002, MNRAS, 335, 1100

Diplas, A., \& Savage, B. D. 1994, ApJ, 427, 274

Dumm, T., Schmutz, W., Schild, H., \& Nussbaumer, H. 1999, A\&A, 349, 169

Fekel, F. C., Joyce, R. R., Hinkle, K. H., \& Skrutskie, M. 2000, AJ, 119, 1375

Fernández-Castro, T., González-Riestra, R., Cassatella, A., Taylor, A. R., \& Seaquist E. R. 1995, ApJ, 442, 366

Fujimoto, M. Y. 1982, ApJ, 257, 767

Gurzadyan, G. A. 1997, The physics and dynamics of planetary nebulae (Berlin Heidelberg: Springer-Verlag)

Heber, U., Napiwotzki, R., \& Reid, I. N. 1997, A\&A,323,819

Hoare, M. G., \& Drew, J. E. 1991, MNRAS, 249, 452

Hummer, D. G., \& Seaton, M. J. 1963, MNRAS, 125, 437

Ignace, R., Cassinelli, J. P., \& Bjorkman, J. E. 1996, ApJ, 459, 671

Kenyon, S. J. 1986, The Symbiotic Stars (Cambridge; CUP)

Kenyon, S. J., \& Webbink, R. F. 1984, ApJ, 279, 252

Kenyon, S. J., Oliversen, N. A., Mikolajewska, J., et al. 1991, AJ, 101, 637

Koester, D., Dreizler, S., Weidemann, V., \& Allard, N. F. 1998, A\&A, 338, 612

Krolik, J. H., \& Raymond, J. C. 1985, ApJ, 298, 660

Lamers, H. J. G. L. M., \& Cassinelli, J. P. 1999, Introduction to stellar winds (Cambridge University Press)

Mikołajewska, J., \& Kenyon, S. J. 1992, AJ, 103, 579

Mikołajewski, M., Mikołajewska, J., Tomov, T., Kulesza, B., \& Szczerba, R. 1990, Acta Astron., 40, 129

Mikołajewska, J., Kenyon, S. J., Mikołajewski, M., Garcia, M. R., \& Polidan, R. S. 1995, AJ, 109, 1289

Mikołajewska, J., Ivison, R. J., \& Omont, A. 2002, Adv. Space Res., 30, 2045

Munari, U. 1992, A\&A, 257, 163

Mürset, U., \& Nussbaumer, H. 1994, A\&A, 282, 586
Mürset, U., Nussbaumer, H., Schmid, H. M., \& Vogel, M. 1991, A\&A, 248, 458

Mürset, U., Wolff, B., \& Jordan, S. 1997, A\&A, 319, 201

Nussbaumer, H., \& Vogel, M. 1987, A\&A, 182, 51

Nussbaumer, H., Schmutz, W., \& Vogel, M. 1995, A\&A, 293, L13

Osterbrock, D. E. 1974, Astrophysics of gaseous nebulae (W. H. Freeman and Company)

Paczyński, B., \& Rudak, R. 1980, A\&A, 82, 349

Popham, R., \& Narayan, R. 1995, ApJ, 442, 337

Schild, H., Dumm, T., Mürset, U., et al. 2001, A\&A, 366, 972

Schmid, H. M., \& Schild, H. 1997b, A\&A, 321, 791

Seaquist, E. R., Taylor, A. R., \& Button, S. 1984, ApJ, 284, 202

Sekeráš, M., \& Skopal, A. 2012a, Baltic Astron., 21, 196

Sekeráš, M., \& Skopal, A. 2012b, MNRAS, in press

Sion, E. M., Huang, M., Szkody, P., \& Cheng, F.-H. 1995, ApJ, 445, L31

Skopal, A. 1998, A\&A, 338, 599

Skopal, A. 2005, A\&A, 440, 995

Skopal, A. 2006, A\&A, 457, 1003

Skopal, A. 2008, JAAVSO, 36, 9

Skopal, A., Vittone, A., Errico, L., et al. 1997, MNRAS, 292, 703

Skopal, A., Djurašević, G., Jones, A., et al. 2000, MNRAS, 311, 225

Skopal, A., Komžík, R., \& Csatáryová, M. 2005, Astrophys. Space Sci., 296, 427

Skopal, A., Vittone, A. A., Errico, L., et al. 2006, A\&A, 453, 279

Skopal, A., Sekeráš, M., González-Riestra, R., \& Viotti, R. F. 2009, A\&A, 507, 1531

Skopal, A., Tarasova, T. N., Cariková, Z., et al. 2011, A\&A, 536, A27

Sokoloski, J. L., Kenyon, S. J., Espey, B. R., et al. 2006, ApJ, 636, 1002

Tutukov, A. V., \& Yangelson, L. R. 1976, Astrofizika, 12, 521

Vrtilek, S. D., Silber, A., Raymond, J. C., \& Patterson, J. 1994, ApJ, 425, 787

Wiecek, M., Mikolajewski, M., Tomov, T., et al. 2010, in Binaries - Key to Comprehension of the Universe, eds. A. Prša, \& M. Zejda (San Francisco, CA: ASP), ASP Conf. Ser., 435, 355 